\title{
On the use of Schur Decomposition for Copyright Protection of Digital Images
}

\author{
B.Chandra Mohan and K.Veera Swamy
}

\begin{abstract}
This paper presents a robust image watermarking scheme for copyright protection of digital images. In this work, Schur decomposition of host image is explored for watermarking . Watermark image is embedded in the two decompositions of Schur decomposition. Schur decomposition is computationally faster and robust to image attacks than Singular Value Decomposition (SVD). Watermark image is embedded in the $D$ component using scalar quantization. A copy of the watermark is embedded in the columns of $U$ matrix using comparison of the coefficients with respect to the watermark image. Since same watermark is embedded in both $U$ matrix and D matrix the robustness is improved. The proposed algorithm is more secure and robust to various attacks, viz., JPEG2000 compression, JPEG compression, rotation, scaling, cropping, row-column blanking, row-column copying, salt and pepper noise, filtering and gamma correction. Superior experimental results are observed with the proposed algorithm over a recent scheme proposed by Chung et al. in terms of Normalized Cross correlation (NCC) and Peak Signal to Noise Ratio (PSNR).
\end{abstract}

Index Terms-Schur decomposition, SVD decomposition, PSNR, and NCC.

\section{INTRODUCTION}

The ability to hide information or data without invoking suspicion is required in many applications, viz., copy right protection, data authentication, and security, which is having application in defense and military. These techniques are well known under terms of 'Cryptography', 'Steganography', and 'Watermarking'. Cryptography is about protecting the content of messages (their meaning), steganography is about concealing their very existence. The purpose of steganography is having a covert communication between two parties whose existence is unknown to a possible attacker. A successful attack consists in detecting the existence of this communication (e.g., using statistical analysis of images with and without hidden information). Watermarking, as opposed to steganography, has the (additional) requirement of robustness against possible attacks. The process of embedding multimedia information into another object/signal can also be termed as watermarking. In watermarking, a watermark (image, PN sequence or audio) is inserted into the cover signal, viz., image, audio or video in an unperceivable manner and accordingly they are known

Manuscript received December 26, 2009.

B.Chandra Mohan is with ECE department, Bapatla Engineering College, Bapatla. He can be reached at chandrabhuma@gmail.com.

K.Veera Swamy is with ECE department, QIS College of Engineering and Technology, Ongole. He can be reached at kilarivs@yahoo.com. as image, audio, and video watermarking respectively.

Watermarking can be used to insert digital object identifier or serial number to help archive digital contents like images, audio or video. Normally digital contents are identified by their file names; however, this is a very fragile technique as file names can be easily changed. Hence, embedding the object identifier within the object itself reduces the possibility of tampering and hence can be effectively used in archiving systems. This is an important characteristic of watermarking, an additional advantage compared to other data hiding techniques, viz., cryptography and steganography.

Digital image watermarking algorithms available in the current literature are categorized into spatial, transform, and hybrid domain techniques. In spatial domain watermarking algorithms, the pixel intensities of the cover image are modified as per the watermark bits. These algorithms are simple, computationally less expensive, and more data can be embedded. However, these algorithms are not preferred as they are not robust to many image attacks and insecure.

In transform domain based watermarking algorithms, an orthogonal transform is applied to the cover image and is transformed into another domain. The transform coefficients of the cover image are modified as per the watermark bits. Transform domain based watermarking algorithms are secure and robust to many image attacks. However, more data cannot be embedded in the transform domain. Several transform domain based (DCT, DHT, SVD, DWT, and CT) $[1,2,3,4,5,6,7,8,9,17]$ watermarking algorithms are available in the current literature. However, these algorithms fail to achieve all requirements of digital image watermarking system, viz., imperceptibility of watermark image, robustness against attacks, information hiding capacity, security, successful retrieval of watermark, and reduced computational complexity.

In hybrid domain watermarking algorithms, both the domains (spatial and transform) are explored for watermark embedding. One major requirement of watermarking systems is that the embedded watermark should survive even after common (and sometimes uncommon) image attacks namely, JPEG compression, rotation, scaling, etc. There are several image attacks listed in the literature. Checkmark [11], Optimark and Stirmark are three popular benchmarking tools used for assessing the robustness of the watermarking system. As per the available literature it is understood that not much emphasis is given on usage of these tools while assessing the robustness. In this work, Checkmark is used for assessing the robustness of the proposed watermarking system. This paper is organized as follows. Schur decomposition is discussed in 
section II. Scalar quantization is presented in section III. Proposed algorithm is explained in section IV. Experimental results and concluding remarks are given in section V and VI respectively.

\section{SCHUR DECOMPOSITION}

Schur decomposition [14] of a real matrix $A$ results in two matrices $U$ and $D$ such that

$$
A=U \times D \times U^{\prime}
$$

Heres $D$ is an upper triangular matrix. $U$ is a unitary matrix. $U^{\prime}$ indicates transpose of $U$. $D$ has the real eigenvalues on the diagonal and the complex eigenvalues in 2-by-2 blocks on the diagonal. Schur decomposition requires about $\frac{8}{3} N^{3}$ flops. This is less than one third the number of computations required for SVD decompositions that require about $11 N^{3}$ flops. In Schur decomposition, the matrix $U$ has one interesting property, i.e. all its first two column elements are of same sign and their values are very close. This property can be explored for image watermarking. For illustration, for a sample matrix

$$
A=\left[\begin{array}{llll}
160 & 160 & 160 & 159 \\
161 & 160 & 159 & 159 \\
160 & 161 & 160 & 158 \\
161 & 161 & 159 & 159
\end{array}\right]
$$

Schur decomposition of $A$ produces $U$ and $D$ matrices

$$
\begin{aligned}
& U=\left[\begin{array}{cccc}
-0.4998 & 0.2448 & 0.7835 & 0.2765 \\
-0.4998 & -0.2843 & 0.0580 & -0.8161 \\
-0.4998 & 0.6754 & -0.5413 & 0.0323 \\
-0.5006 & -0.6349 & -0.2998 & 0.5065
\end{array}\right] \\
& D=\left[\begin{array}{cccc}
639.2483 & -1.1901 & -2.2823 & 2.0916 \\
0 & 0.8139 & -1.0125 & -0.6003 \\
0 & 0 & -0.5311 & 0.9011 \\
0 & 0 & -0.2695 & -0.5311
\end{array}\right]
\end{aligned}
$$

The sign of the $U$ matrix column elements is same (negative) . This is further verified by considering another sample matrix

$$
A=\left[\begin{array}{cccc}
161 & 29 & 180 & 23 \\
160 & 165 & 164 & 23 \\
10 & 89 & 167 & 120 \\
23 & 44 & 127 & 122
\end{array}\right]
$$

$$
U=\left[\begin{array}{cccc}
-0.4620 & -0.3885 & -0.7311 & -0.3178 \\
-0.6707 & 0.7212 & -0.0334 & 0.1701 \\
-0.4593 & -0.2728 & 0.6631 & -0.5243 \\
-0.3546 & -0.5045 & 0.1568 & 0.7715
\end{array}\right]
$$

As can be seen from $U$ matrix, all the first two column elements are of same sign and difference of first two column elements is very small. In schur decomposition, there exists a strong correlation between the first row first column elementof and second row first column element. A matrix consisting of first row first column element of each schur decomposed $U$ matrix block and another one consisting of second row first column element of each schur decomposed $U$ matrix block is formed. Normalized cross correlation between the two matrices is calculated and is listed in Table 1. For majority of the images, this value is very close to 1 . For standard test images this value is computed and given below. For comparison, when SVD is used, the correlation value is very low compared to Schur decomposition shown in the Table 1.

$$
D=\left[\begin{array}{cccc}
399.6806 & 94.0232 & -80.1419 & 100.2003 \\
0 & 89.6390 & -84.4237 & -36.5183 \\
0 & 32.9343 & 89.6390 & 108.4727 \\
0 & 0 & 0 & 36.0414
\end{array}\right]
$$

TABLE 1: NCC OF SVD AND SCHUR DECOMPOSITION

\begin{tabular}{|l|l|l|l|}
\hline Image & $\begin{array}{l}\text { NCC of } \\
\text { SVD } \\
U \text { matrix }\end{array}$ & $\begin{array}{l}\text { NCC of } \\
\text { SVD } \\
V \text { Matrix }\end{array}$ & $\begin{array}{l}\text { NCC of } \\
\text { Schur } \\
U \text { matrix }\end{array}$ \\
\hline Lena & 0.9620 & 0.9533 & 0.9995 \\
\hline Barbara & 0.9691 & 0.9362 & 0.9989 \\
\hline Aeroplane & 0.9496 & 0.9695 & 0.9996 \\
\hline Tank & 0.8903 & 0.9083 & 0.9991 \\
\hline Elaine & 0.9690 & 0.9770 & 0.9755 \\
\hline Boat & 0.9376 & 0.8934 & 0.9969 \\
\hline Mandrill & 0.6436 & 0.8912 & 0.9934 \\
\hline
\end{tabular}

\section{SCALAR QUANTIZATION}

There are several scalar quantization schemes available in the literature for watermarking applications. But, the scalar quantization, known as dither quantization proposed by Chen and Wornell [10] is used in this work for watermark embedding. Dither quantization is a variant of QIM [10]. Dither quantizers are quantizer ensembles. Each quantization cell in the ensemble is constructed from a basic quantizer. The basic quantizer may be chosen arbitrarily. However, the basic quantizer chosen here is a uniform quantizer.

The basic quantizer is shifted to get the reconstruction point. The shift depends on the watermark bit. The basic quantizer is a uniform scalar quantizer with a fixed step size. Even though, step size is fixed for an image, it varies from image to image. Proper value of step size has to be selected based on some experimentation. The quantized value is the center of the quantizer. Dither quantization of an image 
$h(i, j)$ is described as follows:

The entire range $h_{\min }$ (minimum value of $\left.h(i, j)\right)$ to $h_{\max }$ (maximum value of $h(i, j)$ ) is divided into various bins as shown in Table 2. A step size of $\mathrm{T}$ is taken as the difference from one bin to another bin. Each element of $h(i, j)$ is checked for its position in Table 2 .

TABLE 2: QUANTIZATION TABLE
\begin{tabular}{|l|l|l|}
\hline $\begin{array}{c}\text { bin } n o \\
(n)\end{array}$ & \multicolumn{1}{|c|}{ Dlow } & \multicolumn{1}{c|}{ dhigh } \\
\hline 1 & $h_{\min }-\mathrm{T}$ & $h_{\min }$ \\
\hline 2 & $h_{\min }$ & $h_{\min }+\mathrm{T}$ \\
\hline 3 & $h_{\min }+\mathrm{T}$ & $h_{\min }+2 \mathrm{~T}$ \\
\hline$\cdot$ & $\cdot$ & $\cdot$ \\
. &. & $\cdot$ \\
\hline$b_{n-1}$ & $h_{\max }-\mathrm{T}$ & $h_{\max }$ \\
\hline$b_{n}$ & $h_{\max }$ & $h_{\max }+\mathrm{T}$ \\
\hline
\end{tabular}

After identifying the bin number $n, h(i, j)$ is modified as follows:

(i) If watermark bit is ' 1 ' then it belongs to Range 1 where Range 1 is defined as

$$
\text { Range } 1=\operatorname{dlow}(n) \text { to } \frac{\operatorname{dlow}(n)+\operatorname{dhigh}(n)}{2}
$$

Modification of $h(i, j)$ is

$$
h(i, j)=\left(\frac{(\operatorname{dlow}(n)+(\operatorname{dlow}(n)+\operatorname{dhigh}(n)) / 2}{2}\right)
$$

(ii) If watermark bit is ' 0 ' then it belongs to Range 2 where Range 2 is defined as

$$
\text { Range } 2=\frac{\operatorname{dlow}(n)+\operatorname{dhigh}(n)}{2} \text { to } \operatorname{dhigh}(n)
$$

Modification of $h(i, j)$ is

$$
h(i, j)=\left(\frac{(\operatorname{dhigh}(n)+(\operatorname{dlow}(n)+\operatorname{dhigh}(n)) / 2}{2}\right)
$$

\section{Proposed Algorithm}

\section{A. Proposed Watermark Embedding Algorithm}

The steps of embedding algorithm are as follows.

- Apply block based Schur decomposition on the grey scale image $f(x, y)$ of size $M \times M$ Size of the block is $n x n$. Two matrices $U_{1}$ and $D_{1}$ of size $M \times M$ are obtained. $U_{1}$ is a unitary matrix and $D_{1}$ is an upper triangular matrix.

- $\quad$ The elements of $D_{1}$ matrix are modified (quantized) using scalar quantization using Eqs.(3) and (4).

- Inverse Schur decomposition is applied, i.e., on modified $D$ and unmodified $U$ matrices.

$$
A_{\text {wat } 1}=U_{1} \times D_{\text {mod }} \times D_{1}^{\prime}
$$

Here, $D_{1}^{\prime}$ indicates transpose of $D_{1}$.
- Apply block based Schur Decomposition on $A_{\text {wat } 1}$ which results in two matrices $U_{2}$ and $D_{2}$.

- A copy of watermark is embedded in the $U_{2}$ matrix using the following formula For each $n x n$ block of $U_{2}$ matrix, $u_{11}$ (first row, first column) and $u_{21}$ (second row, first column) are modified as follows:

There is no change in $u_{11}$ and $u_{21}$ provided

$$
\begin{aligned}
& w(i, j)=1 \& u_{11}>u_{21} \\
& \text { or } \\
& w(i, j)=0 \& u_{11}<u_{21}
\end{aligned}
$$

Otherwise, two elements $u_{11}$ and $u_{21}$ are swapped. The coefficients $u_{11}$ (first row, first column) and $u_{21}$ (second row, first column) are selected for watermark embedding because their strong correlation across all the blocks (of same sign) and the difference between the two values is very small.

- Inverse Schur decomposition is applied on the modified $U_{2}$ matrix and unmodified $D_{2}$ matrix. The resultant watermarked image is given by

$$
A_{\text {wat } 2}=U_{2 \mathrm{mod}} \times D_{2} \times U_{2 \mathrm{mod}}{ }^{\prime}
$$

where, $U_{2 \bmod }$ ' indicates transpose of $U_{2 \bmod }$.

The steps of watermark extraction are as follows.

- Apply Schur decomposition on the watermarked image $A_{\text {wat } 2}$.

- From the resulting $D_{2}$ matrix extract the watermark $w(i, j)$ using scalar quantization.

- From the resulting $U_{2 \bmod }$ matrix extract the watermark as per the following If $u_{11}>u_{21}$ then, $w(i, j)=1$, else $w(i, j)=0$.

In this way, watermark is extracted in two steps from both $U_{2 \text { mod }}$ and $D_{2}$ matrices. If the extraction is not perfect, a small incremental change in the coefficients may result in perfect extraction.

\section{EXPERIMENTAL RESULTS}

To test the robustness of the proposed scheme, experiments are conducted on several images of benchmark image database [16]. These images are listed in Table 3. However, the results for various attacks are presented by considering cover image 'Lena' as shown in Fig.1(a). The size of the cover image is $512 \times 512$. The watermark image is of $32 \times 32$ size which is a logo 'JNTU' as shown in Fig.1(b). Step size in the scalar quantization is selected in such a way that the watermark extraction is perfect. It is taken as 60 for the experimentation. Imperceptibility of the watermark image in terms of PSNR is $43.05 \mathrm{~dB}$. In addition to PSNR, other quality metrics $[13,15]$ are also used to assess the quality of the watermarked image and are listed in Table 3. From Table 3, it can be inferred that the quality of the 
watermarked is good .

All the attacks, except image tampering and JPEG2000 attack, are tested using MATLAB 7.0. JPEG2000 attack is tested using 'Morgan JPEG2000 tool box' [12] and image tampering is done with 'Paintbrush'. Various attacks used to test the robustness of the watermark are JPEG2000, JPEG compression, rotation, resizing, low pass filtering, median filtering, cropping, row column blanking, row column copying, salt \& pepper noise, bit plane removal, gamma correction, and image tampering. The extracted watermarks after applying various attacks are summarized in Fig. 2. Rotation is a lossy operation. The watermarked image is rotated by $15^{0}$ to the right and then rotated back to their original position using bilinear interpolation.

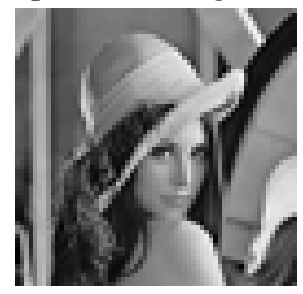

Fig.1(a) Cover Image Lena

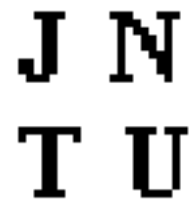

(b) Watermark
For low pass filtering attack, a $3 \times 3$ mask consisting of an intensity of $1 / 9$ is used. The $3 \times 3$ kernel of the lowpass filter is given by

\section{$0.1111 \quad 0.1111 \quad 0.1111$ \\ 0.11110 .11110 .1111 \\ $0.1111 \quad 0.1111 \quad 0.1111$}

When median filtering is applied to watermarked image, each output pixel in the attacked image contains the median value in the 3-by-3 neighborhood around the corresponding pixel in the input image.

Resizing operation first reduces or increases the size of the image and then generates the original image by using an interpolation technique. This operation is a lossy operation and hence the watermarked image also looses some watermark information. In this experiment, first the watermarked image is reduced from $512 \times 512$ size to $256 \times 256$. By using bicubic interpolation, its dimensions are increased to $512 \times 512$. The watermarked image is compressed using lossy JPEG compression.

\section{$0.1111 \quad 0.1111 \quad 0.1111$$$
0.1111 \quad 0.1111 \quad 0.1111
$$$$
0.1111 \quad 0.1111 \quad 0.1111
$$

When median filtering is applied to watermarked image, each output pixel in the attacked image contains the median value in the 3-by-3 neighborhood around the corresponding pixel in the input image.

Resizing operation first reduces or increases the size of the image and then generates the original image by using an interpolation technique. This operation is a lossy operation and hence the watermarked image also looses some watermark information. In this experiment, first the watermarked image is reduced from $512 \times 512$ size to 256x256. By using bicubic interpolation, its dimensions are increased to $512 \times 512$. The watermarked image is compressed using lossy JPEG compression.

The index of the JPEG compression ranges from 0 (best compression) to 100 (best quality). The proposed scheme works well even for 50\% compression. Similarly, JPEG2000 compression is used to test the robustness with varying quality factor. The results are good indicating that the proposed method is able to survive even after JPEG2000 compression. The watermarked image is attacked by salt \& pepper noise with a noise densities of 0.001 and 0.003 . All the extracted watermarks are clearly visible indicating the proposed method's resilience to noise attack.

In row column blanking attack, a set of rows and columns are deleted.In this experiment, 10,30,40,70,100,120, and 140 numbered rows and columns are removed.

In row-column copy attack, a set of rows and columns are copied to the adjacent or random locations. In this attack, $140^{\text {th }}$ row is copied to $160^{\text {th }}$ row.

Histogram equalization is a technique used in image processing to enhance images. In this work, the histogram of the input image is modified in such a way that the processed image histogram is nearly uniform. The watermark image is usually lost in the histogram equalization attack. The watermark is easily recognizable even after histogram equalization attack.

\begin{tabular}{|c|c|c|}
\hline $\begin{array}{l}\text { Attack type \& } \\
\text { PSNR of the } \\
\text { watermarked } \\
\text { image }\end{array}$ & $\begin{array}{c}\text { Extracted } \\
\text { Watermark from } \\
U \text { matrix }\end{array}$ & $\begin{array}{l}\text { Extracted Watermark } \\
\text { from } D \text { matrix }\end{array}$ \\
\hline \multicolumn{3}{|l|}{$\begin{array}{c}\text { Tampering } \\
27.6115\end{array}$} \\
\hline \multicolumn{3}{|l|}{$\begin{array}{c}\text { Contrast } \\
\text { Enhancement } \\
(50 \%) \\
20.6852\end{array}$} \\
\hline \multicolumn{3}{|l|}{$\begin{array}{c}\text { Aspect Ratio } \\
\text { Change } \\
34.0816\end{array}$} \\
\hline $\begin{array}{c}\text { JPEG2000 } \\
42.5493\end{array}$ & & \\
\hline & 0.8860 & 1.0000 \\
\hline
\end{tabular}




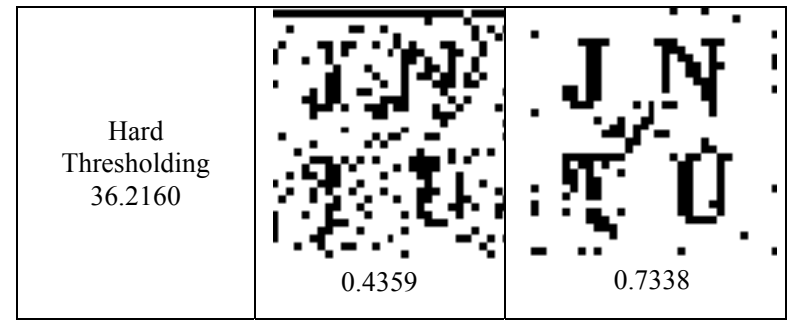

Fig 2. Extracted Watermarks (contd..)

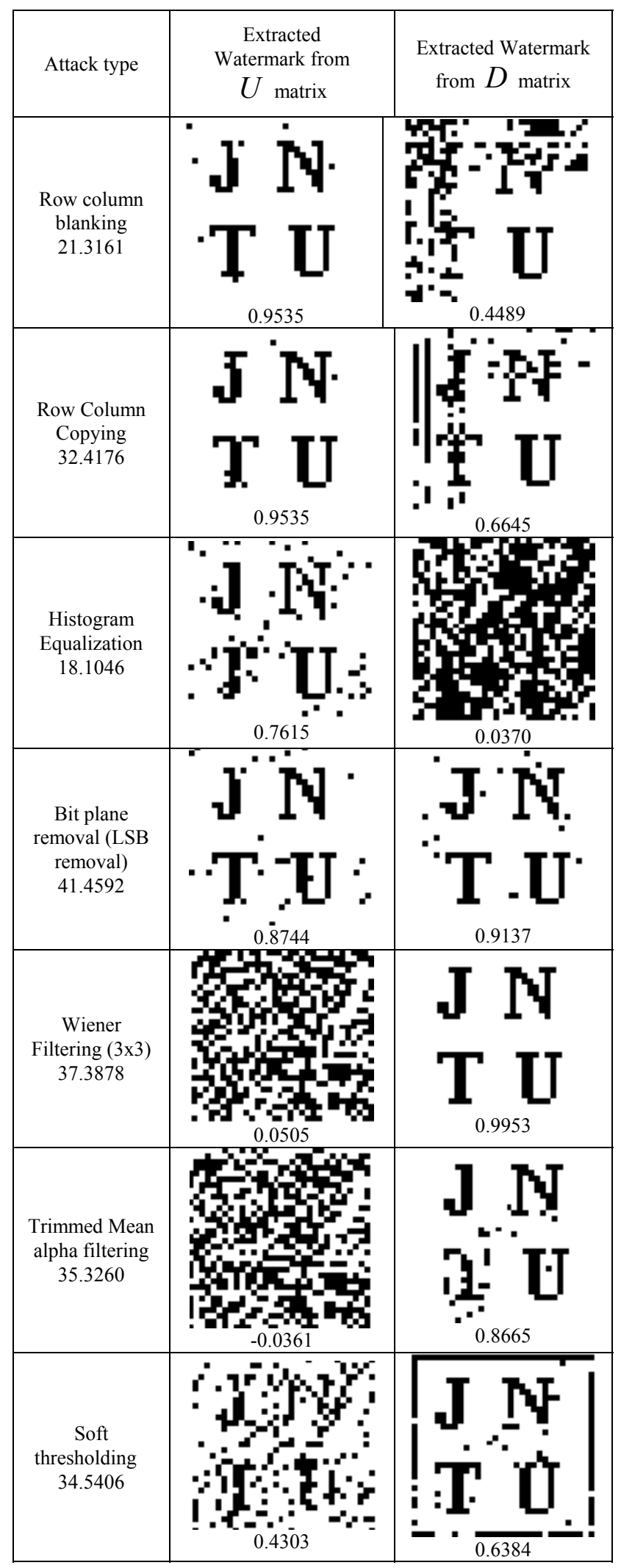

Fig. 2 Extracted Watermarks (contd..)

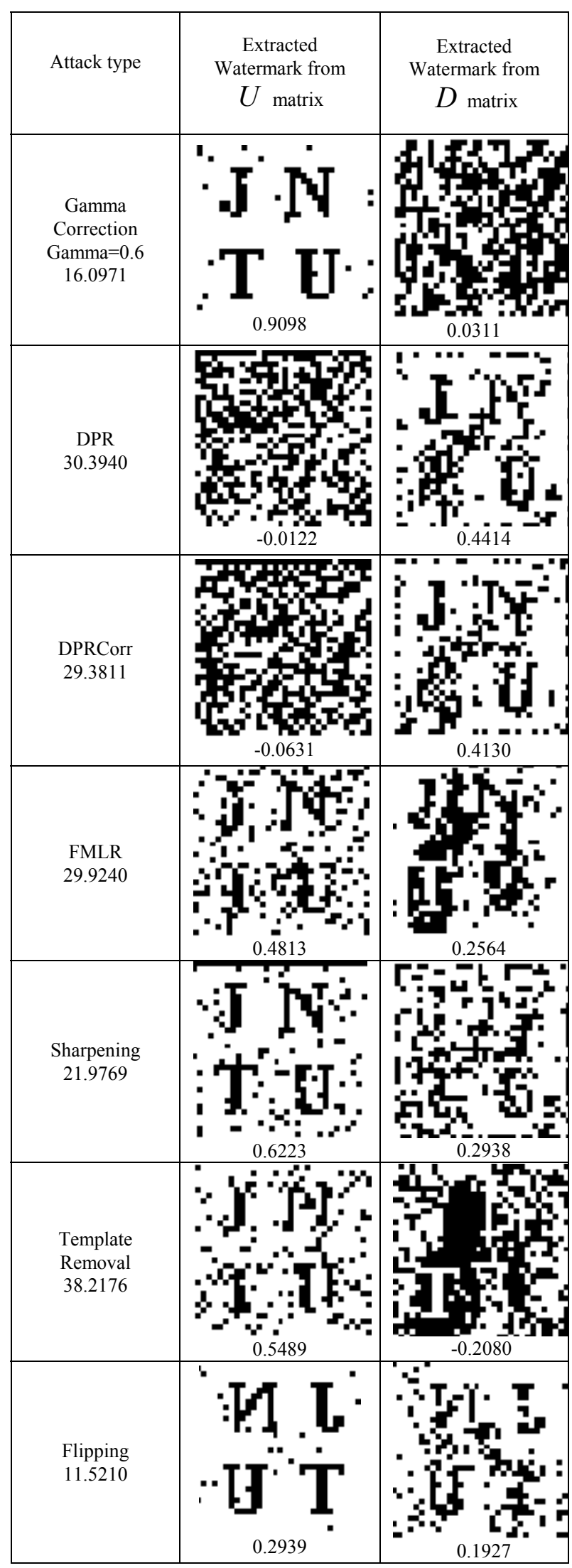

Fig. 2 Extracted Watermarks (contd..) 
TABLE 3: QUALITY ASSESSMENT BETWEEN THE ORIGINAL AND WATERMARKED IMAGES

\begin{tabular}{|c|c|c|c|c|c|c|c|}
\hline \multirow[b]{2}{*}{ Quality Metric } & Lena & Barbara & Aeroplane & Tank & Elaine & Boat & Mandrill \\
\hline & & & & & & & \\
\hline $\begin{array}{l}\text { Mean Square } \\
\text { Error(MSE) }\end{array}$ & 3.218479 & 3.914845 & 3.378674 & 3.765301 & 3.430950 & 5.122185 & 15.874619 \\
\hline $\begin{array}{l}\text { Peak Signal to } \\
\text { Noise Ratio } \\
\text { (PSNR in dB) }\end{array}$ & 43.054297 & 42.203658 & 42.843341 & 42.372807 & 42.776660 & 41.036251 & 36.123771 \\
\hline $\begin{array}{l}\text { AD(Average } \\
\text { Difference) }\end{array}$ & 0.646420 & 0.640308 & 0.601414 & 0.708920 & 0.597546 & 0.708138 & 0.955170 \\
\hline $\begin{array}{l}\text { Structural } \\
\text { Content }\end{array}$ & 1.008579 & 1.009116 & 1.006584 & 1.010272 & 1.007480 & 1.009892 & 1.010759 \\
\hline $\begin{array}{c}\text { NK(Normalized } \\
\text { Cross correlation) }\end{array}$ & 0.995656 & 0.995366 & 0.996676 & 0.994813 & 0.996205 & 0.994968 & 0.994316 \\
\hline $\begin{array}{l}\text { MD(Maximum } \\
\text { Difference) }\end{array}$ & 55.000000 & 75.000000 & 52.000000 & 53.000000 & 48.000000 & 123.000000 & 136.000000 \\
\hline $\begin{array}{l}\text { LMSE(Laplacian } \\
\text { Mean Square } \\
\text { Errror) }\end{array}$ & 0.036099 & 0.006083 & 0.044623 & 0.027743 & 0.016695 & 0.026733 & 0.020293 \\
\hline $\begin{array}{c}\text { Normalized } \\
\text { Absolute Error }\end{array}$ & 0.010277 & 0.010670 & 0.007459 & 0.010415 & 0.009280 & 0.009874 & 0.011610 \\
\hline SSIM & 0.9672 & 0.9836 & 0.9111 & 0.9850 & 0.9834 & 0.9854 & 0.9848 \\
\hline
\end{tabular}

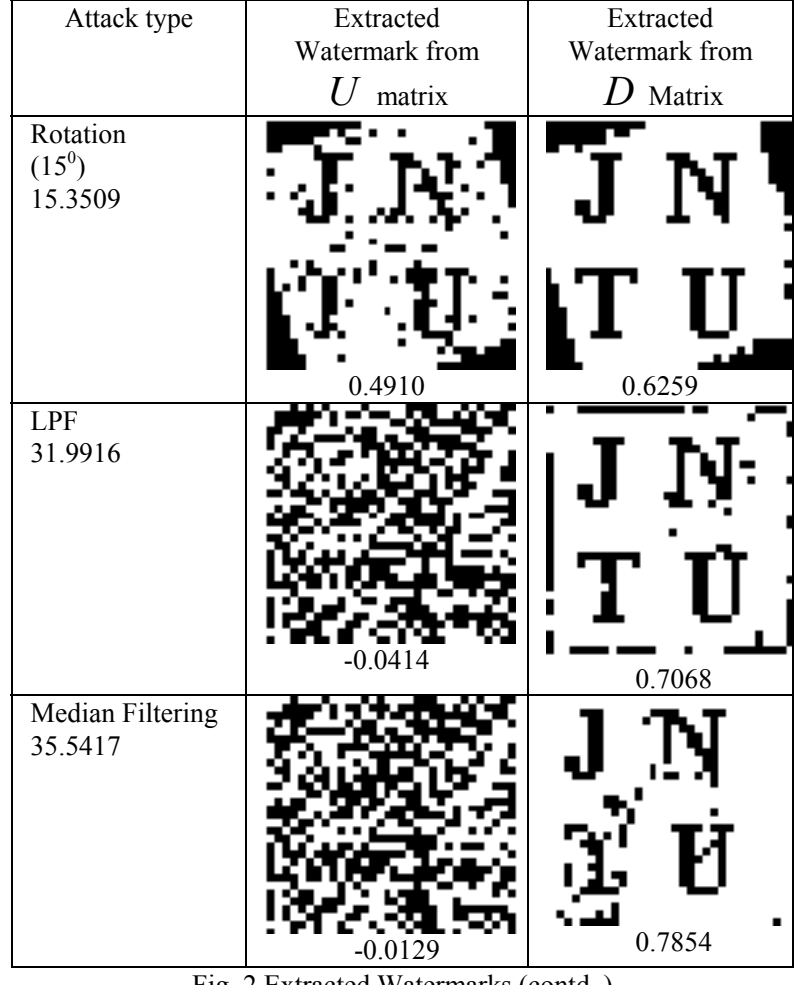

Fig. 2 Extracted Watermarks (contd..)

The proposed algorithm is also resistant to biplane removal. In bit plane removal attack, the least significant bit of the watermarked image is replaced with a zero.

\begin{tabular}{|l|l|l|}
\hline Attack type & Extracted & Extracted \\
& Watermark from & Watermark from $D$ \\
& $U_{\text {matrix }}$ & Matrix \\
\hline
\end{tabular}

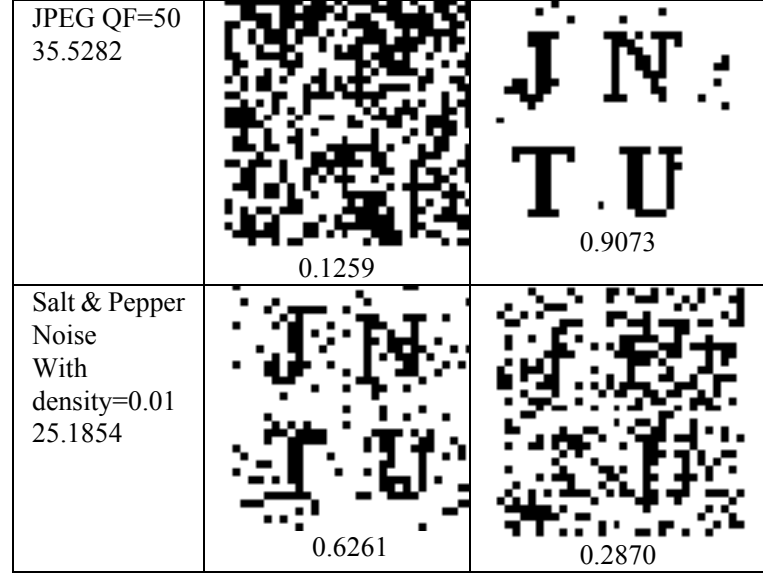

Fig. 2 Extracted Watermarks

In contrast enhancement attack, the contrast of the image changed by $50 \%$. One common image attack while testing the robustness is the survival of the watermark image against noise attacks. The watermarked image is corrupted by salt \& pepper noise with a density of 0.01 .

In gamma correction attack, the watermarked image is mapped to another image by applying a non-linear mapping. Here, gamma specifies the shape of the curve describing the relationship between the input and output. The watermarked image is tampered by writing some 'slogan' on the hat of Lena. Other attacks that are included in Checkmark toolbox are change of aspect ratio, soft thresholding, hard thresholding, Frequency Mode Laplacian Removal, DPR, DPRcorr, template removal, sharpening, brightness enhancement etc.

The proposed algorithm is robust to many image attacks listed in Checkmark toolbox. However, for some image attacks like Collage attack, warping, shearing and projective transformation attacks, the performance of the proposed 
algorithm is not encouraging. Compared to Chung et al., method [18] the proposed is superior both in terms of PSNR and resilience to image attacks. A comparison is outlined in Table 4.

TABLE 4 . COMPARISON OF THE PROPOSED METHOD WITH CHUNG ET.AL.,

\begin{tabular}{|c|c|c|}
\hline \multicolumn{3}{|c|}{ METHOD [18] } \\
\hline Parameter & $\begin{array}{l}\text { Chung et al., } \\
{[18]}\end{array}$ & Proposed Method \\
\hline Transform & SVD & Schur Decomposition \\
\hline $\begin{array}{l}\text { Cover image } \\
\text { Size }\end{array}$ & $512 \times 512$ & $512 \times 512$ \\
\hline $\begin{array}{l}\text { Watermark image } \\
\text { size }\end{array}$ & $32 \times 32$ & $32 \times 32$ \\
\hline $\begin{array}{l}\text { No.of image attacks } \\
\text { reported }\end{array}$ & 3 & 25 \\
\hline $\begin{array}{l}\text { PSNR between cover } \\
\text { image and } \\
\text { watermarked image }\end{array}$ & $38.69 \mathrm{~dB}$ & $43.05 \mathrm{~dB}$ \\
\hline Quality Assessment & $\begin{array}{l}\text { Not } \\
\text { performed }\end{array}$ & Performed \\
\hline
\end{tabular}

\section{CONCLUSIONS}

In this work, a novel watermarking algorithm based on Schur decomposition is proposed. Schur decomposition is computationally faster compared to SVD decomposition and has some important properties (same sign in the column elements of $U$ ) suitable for image watermarking applications. To improve robustness, a watermark is embedded in both $U$ and $D$ matrices of Schur decomposition. Since the proposed scheme is robust to many image attacks, this scheme can be used for copyright protection applications. Compared to an existing method by Chung [18], the proposed method is superior in terms of PSNR of the watermarked image and robustness to image attacks.

\section{REFERENCES}

[1] Chu, W.C., 2003. DCT based image watermarking using sub sampling, IEEE Transactions Multimedia, pp.34-38.

[2] Wang, Y., Alan Pearmain., 2004. Blind image data hiding based on self reference. Pattern Recognition Letters 25, pp. 1681-1689.

[3] Barni, M., Bartolini, M., Piva, F.V., 2001. Improved wavelet based watermarking through pixel-wise masking. IEEE Transactions on Image Processing 10, pp. 783-791.

[4] Lee, C., Lee, H., 2005. Geometric attack resistant watermarking in wavelet transform domain, Optics Express vol.13, no.4, pp.1307-1321

[5] Chandra Mohan B, Srinivas Kumar S, Chatterjee B.N.,2006. Digital Image watermarking in dual domains at IET Visual Information Engineering, VIE 2006 at Leela Palace, Bangalore, 26-28th September 2006.

[6] Santi P.Maity, Malay K Kundu, 2009. DHT domain digital watermarking with low loss in image informations, International Journal of Electronics and Communications, 2009. in press.

[7] Srinivas Kumar S, Chandra Mohan B, Chatterjee B.N., 2007. An oblivious image watermarking scheme using singular value decomposition. IASTED, International conference on Signal and Image Processing, at Honolulu, Hawaii, USA, August 20-22

[8] Yongdong, Wu, 2005. On the Security of an SVD based ownership watermarking. IEEE transactions on Multimedia, vol 7. no.4.

[9] Chandramouli,R.,Graubard Benjamin, M., Richmond Colin, R., 2001. A multiple description framework for oblivious watermarking.
Proceeding of Security and Watermarking and Multimedia contents III, SPIE vol. 4314

[10] Chen, B., Wornell, G.W., 1998. Digital watermarking and information embedding using dither modulation. Proceedings of the IEEE workshop on Multimedia Signal Processing (MMSP-98), Redondo Beach, CA, December.

[11] www.http://watermarking.unige.ch/Checkmark/

[12] Morgan JPEG 2000 Tool Box, http://Morgan-Multimedia.com.

[13] Z.Wang and Alan C.Bowik, 2001. A Universal Image Quality Index. IEEE Signal Processing Letters.

[14] Gene H.Golub, Charles F.Van Loan, 1996, Matrix Computations, John Hopkins University Press, London.

[15] http://sprljan.com/nikola/matlab/qassessment.html

[16] Bench Marking Image Database, http://sipi.usc.edu/database/

[17] B.Chandra Mohan, S.S. Kumar, "Robust digital watermarking scheme using contourlet transform", International Journal of Computer Science and Network Security (IJCSNS), Vol.8, Issue 2, 2008, pp.43-51.

[18] K.L.Chung, W.N.Yang,Y.H.Huang, S.T.Wu, and Y.C.Hsu, "On SVD-based watermarking algorithm", Applied Mathematics and Computation, Vol.188, 2007, pp.54-57

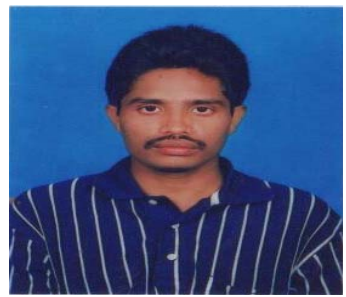

Dr.B.Chandra Mohan is currently working as Professor in ECE Department, Bapatla Engineering College, Bapatla, India. He received his Ph.D from JNTU College of Engineering, Kakinada, India. $\mathrm{He}$ received his M.Tech from Cochin University of Science \& Technolgoy, Cochin, India. $\mathrm{He}$ has 6 international Journal publications to his credit.

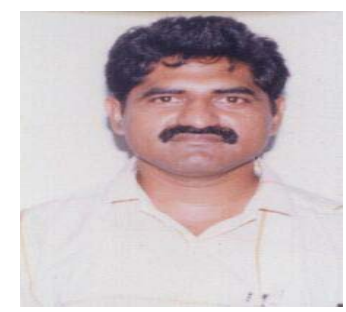

Dr.K.Veera Swamy is currently working as Professor in ECE Department, QIS college of Engineering, Ongole, India. He received his Ph.D from JNTU College of Engineering, Kakinada, India. He received his M.Tech from same college. He has 6 international Journal publications to his credit. 